\title{
Nuevas narrativas en el periodismo actual. El periodismo transmediático
}

\author{
Pilar IRALA HORTAL \\ Universidad San Jorge \\ pirala@usj.es
}

Recibido: 24 de diciembre de 2013

Aceptado: 30 de abril de 2014

\begin{abstract}
Resumen
La evolución de las estrategias de la comunicación en el siglo XXI y la crisis estructural global extendida por el mundo han propiciado el desarrollo de nuevas fórmulas narrativas en el periodismo internacional. Todavía no se pueden considerar líneas prioritarias de comunicación en los medios, pero los jóvenes periodistas comienzan a difundir sus trabajos transmediáticos con gran éxito de lectores y premios. El objetivo de la presente investigación es analizar estas nuevas propuestas periodísticas, sus características y el ejemplo de algunos trabajos destacados. Para ello, se ha repasado la bibliografía nacional e internacional sobre las narrativas transmediáticas y sus particularidades, hemos revisado las aportaciones al tema desde lo teórico y lo práctico y hemos analizado trabajos en medios internacionales.
\end{abstract}

Palabras clave: Periodismo transmediático, discurso audiovisual, narrativa multimedia, cultura digital

\section{New Narratives in the Current Journalism. Transmediatic Journalism}

\begin{abstract}
The evolution in communication strategies in the 21 st century and the global structural crisis which has extended worldwide, has provoked the emergence of new narrative formulas in International journalism. Today these lines cannot be considered priority lines of communication in the media, but young journalists have begun to broadcast their transmedia works with great success among readers and have started to receive awards. The objective of this research is to analyze these new journalistic proposals and show examples of some outstanding works. With this aim in mind we have carried out a literature review of national and international transmedia narrative and their peculiarities have been studied, we have reviewed contributions to the theme from a theoretical and practical perspective and we have analyzed works in international media.
\end{abstract}

Keywords: Transmedia Journalism, Audiovisual Speeches, transmedia storytelling, Digital Culture

\section{Referencia normalizada}

IRALA HORTAL, Pilar (2014): "Nuevas narrativas en el periodismo actual. El periodismo transmediático". Estudios sobre el Mensaje Periodístico. Vol. 20, Núm. 1 (enero-junio), págs.: 147-158. Madrid, Servicio de Publicaciones de la Universidad Complutense.

Sumario: 1. Introducción. 2. Material y métodos. 3. Qué significa "transmediático". 4. Caso de estudio. 5. Conclusiones. 6. Referencias bibliográficas.

\section{Introducción}

Según la definición de Beeson (2005, sin paginar) llamamos narrativa a la diferencia entre la historia y su discurso. Así, la narrativa transmediática es una fórmula de comunicación multimedia e hipertextual basada en la transmisión de un contenido, una historia o pieza periodística a través de diferentes medios (imagen, audio, vídeo) respetando las propias fórmulas de expresión de cada uno de ellos. Se caracteriza, asimismo, por la búsqueda de la participación del lector/espectador quien, al menos, formará parte de la estrategia de difusión de la pieza a través de sus recomendaciones, comentarios o redifusiones. La conceptualización de este fenómeno para el ámbito de 
la narración digital es relativamente reciente así como los primeros estudios sobre la fórmula que fueron comenzadas por Henry Jenkins en 2003. Aunque inicialmente las investigaciones se centraron en la narrativa de ficción (cine y televisión), ya existen estudios que analizan su transferencia al periodismo y piezas de ámbito internacional creadas desde la postura multimediática y transnarrativa. Pero, a pesar de que han pasado algunos años desde que Carlos A. Scolari afirmara que faltaba un importante camino intelectual y analítico por recorrer en el tema de la narrativa transmedia $(15 / 05 / 2009)$, lo cierto es que apenas se ha comenzado a profundizar.

Así, la presente investigación resume las características de la narrativa transmediática que diferentes autores han propuesto y describe cómo aparecen en el periodismo, creando nuevas fórmulas comunicacionales en el entorno digital y su aportación a la democratización de la información. Tras la reflexión especulativa y a partir de los rasgos de la narración transmediática analizaré el trabajo transmediático Spain gets angry de Pablo Zurita y Ofelia de Pablo para The Guardian (20/11/2011).

\section{Material y métodos}

Se ha revisado el material bibliográfico, tanto en lengua española como en lengua inglesa, de los principales autores que han trabajado sobre las nuevas fórmulas narrativas en las últimas décadas, así como los teóricos de la narrativa transmediática. Se ha hecho un vaciado desde los arranques semióticos con Henry Jenkins hasta sus implicaciones en las nuevas fórmulas periodísticas. Asimismo, se han analizado diferentes piezas transmediáticas que cumplían con las características descritas en la literatura analizada y, además, habían sido publicadas en medios internacionales. Posteriormente se analiza la pieza Spain gets angry que contiene información audiovisual y fotoperiodística.

\section{Qué significa "transmediático"}

La revisión de la bibliografía nos lleva a plantear que el mismo término "transmedia" se refiere a dos subgéneros narrativos diferentes cuando hablamos de ficción o de no ficción. En los productos de ficción el vocablo se refiere a la difusión de contenidos de ese producto a través de diferentes medios: tv, internet, comic, videojuegos, libros...Cada uno de esos medios o plataformas aportan novedades narrativas al conjunto, es decir, se puede ver un capítulo de la serie en la televisión, pero en el videojuego la acción continua de otra forma y así sucesivamente. En este caso, el espectador/lector/jugador es también guionista.

Pero cuando nos referimos a la no ficción el término parece referirse más estrictamente al uso de diferentes lenguajes para comunicar de una manera más profunda y completa acerca de un hecho: el caso que aquí analizamos es un ejemplo. Pero también nos encontramos con piezas muy interesantes de narrativas de no ficción, sobre todo de reportajes, que usan el cross-media o multiplataforma. Es el caso de Snow Fall (Branch, 2012) publicado en The New York Times. Se trata de un reportaje multimedia, hipertexto y de gran peso visual que narra la historia de una tragedia en la nieve. Contiene mapas, cortes de audio, fotografías y un diseño web adaptado al acontecimiento. 


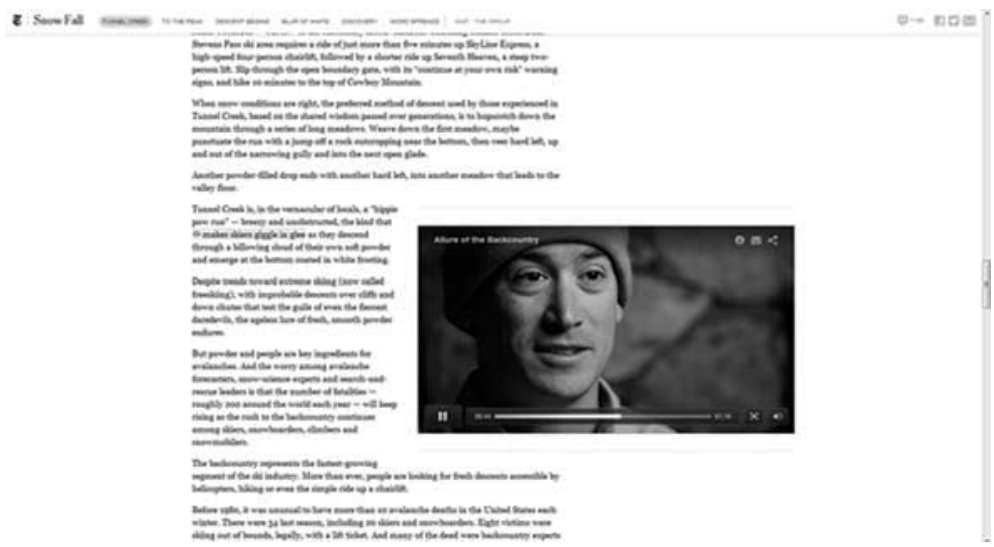

Ilustración 1. Captura de pantalla: inserción de vídeo en la pieza transmediática Snow Fall

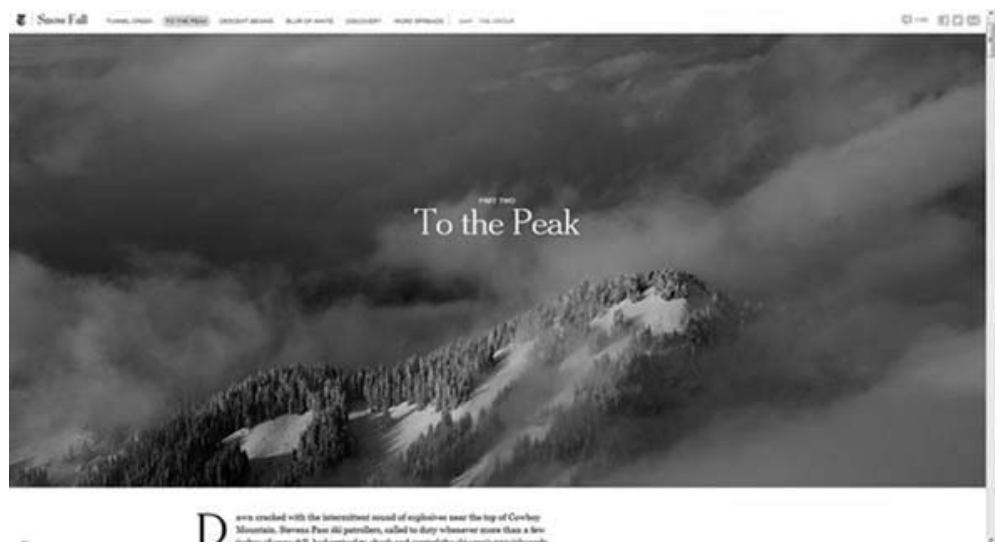

Ilustración 2. Cabecera de un capítulo de la pieza Snow Fall

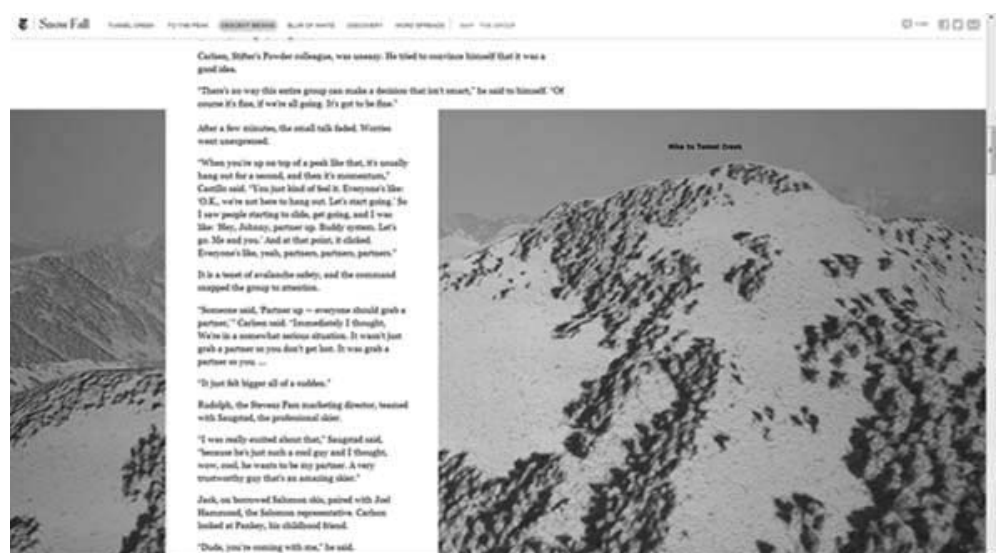

Ilustración 3. Mapa de la travesía narrada en Snow Fall 
Fue Henry Jenkins quien trasladó el término "transmedia" al ámbito de la narrativa audiovisual de no ficción en 2009 y, posteriormente, otros teóricos han aportado nuevas características o han acotado las de Jenkins. Siete son las particularidades de los productos transmedia para Jenkins. Las expuso en un artículo de 2009 continuación de otros escritos sobre el mismo tema como el titulado Transmedia Storytelling 101 (2007) en el que ya planteaba algunas cuestiones básicas como la sistematización en la difusión de estas piezas en las diferentes plataformas, así como la importancia del esfuerzo en coordinarlas; las sinergias con la industria mediática o las diferentes aportaciones de cada plataforma. Posteriormente publicó sus siete principios de la narrativa transmediática para la ficción (2009).

El otro teórico más destacado que se ha acercado a las producciones transmediáticas en el ámbito de la no ficción es Kevin Moloney, quien defendió su tesis doctoral sobre transmedia journalism en 2011. En esta investigación analiza las características aportadas por Jenkins desde la perspectiva periodística. Y continúa explicando cómo algunas de las ideas aportadas por Jenkins tienen un significado diferente cuando hablamos de periodismo transmedia y que, además, ya se desarrollaban antes de que la industria cinematográfica y televisiva lo hiciera (Moloney, 2011: 60). De hecho, pone el ejemplo de la pieza Bosnia: Uncertain Paths to Peace (Peress y Ritchin, 1996)

Moloney presenta sus investigaciones a través de la web http://transmediajournalism.org. En esta plataforma explica y defiende los rasgos e importancia de las fórmulas transmediáticas en el nuevo periodismo. En noviembre de 2011 publicó un breve manifiesto para el periodismo transmediático:

"We journalists need to find the public across a very diverse mediascape rather than expecting them to come to us. The days of the captive journalism audience are over, and if we hope to serve our ideals of democracy, human rights, environment and positive social change, we need to find a broad public. To make our stories salient we need to engage the public in ways that fit those particular media. We lose an opportunity to reach new publics and engage them in different ways when we simply repurpose the same exact story for different (multi) media. Why not use those varying media and their individual advantages to tell different parts of very complex stories? And why not design a story to spread across media as a single, cohesive effort?" (Moloney, 23/11/2011)

De este texto se desprende la importancia que este periodismo le da al factor social y humano, no solo en sus temas, sino también en el interés mostrado por llegar a la mayor cantidad de lectores posible. Tal y como explica Moloney, es un gran universo de posibilidades donde es el periodista quien escoge el medio y pone límites (6/11/2012). La posibilidad de elección entre audio, video, foto fija o un sinfín de posibilidades multimedia permite al periodista usar la mejor fórmula narrativa para cada parte de su pieza, de su investigación. De la misma manera, el lector accede a una ingente cantidad de información que le permite la inmersión en el tema de una forma revolucionaria. En este sentido, es importante que tanto el responsable de la narración como el lector/usuario compartan los mismos códigos narrativos y que estén "bien versados" como indica Beeson (2005, sin paginar). 
Como ya sucede con otros géneros o fórmulas narrativas más tradicionales, como el fotoperiodismo, el periodismo multimedia busca nuevas vías de difusión. Si la fotografía de prensa ha salido de los periódicos y ahora no es raro ver exposiciones de grandes fotorreporteros en salas de exposiciones y museos, también el periodismo de narrativas transmediáticas, por su propia naturaleza, sale de la prensa y lo encontramos en las webs de los medios, museos, encuentros internacionales, blogs especializados (muchas veces de los propios periodistas), pero también buscan en otras vías a sus lectores como en videojuegos o proyecciones públicas (Moloney, 6/11/2012).

Finalmente, se trata de una nueva forma de hacer periodismo, que busca la conexión y la connivencia del lector, así como la mayor inmersión posible en la historia. Estos rasgos ya habían aparecido en otros géneros de la profesión en el XIX. El Periodismo Literario o periodismo de autor, ya había tomado de otras ramas de la comunicación, como la literatura, herramientas creativas para llegar al lector, hacer calar la historia, involucrar a través de las palabras y ahondar en el contexto. En el siglo XXI autores como Jorge Carrión (8/5/2013) profundizan en las nuevas fórmulas que adopta el periodismo para contener y difundir una historia. Se trata de uniones como el periodismo con el comic o el videojuego.

Asimismo, estas ramificaciones o subgéneros narrativos tienen otra cuestión básica en común: los temas que abordan. Todo ellos se basan en largas investigaciones que incluyen profundas documentaciones, inmersiones en el contexto del acontecimiento, diferentes puntos de vista, una historia principal salpicada de subhistorias y, en general, lo que Kramer definió como las “Reglas quebrantables para periodistas literarios” (2001).

Desde diferentes reflexiones, autores y periodistas se llega por diferentes caminos a la misma conclusión: las nuevas fórmulas narrativas han llegado a la profesión y estamos ante el inicio de una nueva era del periodismo, su producción, su consumo y su influencia social.

\section{Caso de estudio}

En este apartado se estudia la pieza Spain gets angry, de Javier Zurita y Ofelia de Pablo (20/06/2011). Se toman como referencia las reflexiones que hace Moloney, donde transporta las características de Jenkins al periodismo (Moloney, 2011: 60 y ss.).

Se trata de una pieza audiovisual que combina diferentes fórmulas narrativas: vídeo, fotografía y audio, así como distintos géneros periodísticos: entrevista, reportaje, documental y fotoperiodismo. Se centra en las protestas ciudadanas contra los recortes del gobierno español, así como en el alto paro del país. Tiene una duración de siete minutos y medio y tiene tres protagonistas que explican sus razones para apoyar al movimiento $15 \mathrm{M}$ y salir a la calle a manifestarse.

A continuación listamos los rasgos de la narrativa transmediática que Moloney traslada al periodismo y las analizamos a la luz del ejemplo propuesto:

- Spreadable (de amplia o fácil difusión): Se refiere a los medios y periódicos que albergan blogs y tienen cuentas en redes sociales. También periodistas que tienen blogs profesionales diferenciados de las cuentas oficiales del medio para el que trabajan y, por supuesto, a las opciones de compartir la noticia entre los lectores, enviar por mail, Facebook, twitter u otras formas de conexión social. Estas opciones abren la comu- 
nicación en varias direcciones y no solo el tradicional sentido único de comunicación: del medio al lector.

"Visits to the Web sites of nearly any legacy and new media outlet show buttons to share stories and links on social media sites, email, SMS or a blog with a single click. Tracking systems such as the New York Times' listings of "Most E-mailed," "Most Viewed" and "Most Blogged" encourage the dissemination of work through the networked sphere by engaging our social instincts further" (Moloney, 2011: 64)

El caso aquí estudiado se publicó en The Guardian (http://www.guardian.co.uk). Este periódico incluye en su versión digital diferentes fórmulas para la conexión con sus lectores. Además de la suscripción online, tienen hemeroteca digital, servicios por correo electrónico, página en Facebook (https://www.facebook.com/theguardian) y cuenta de Twitter (@theguardian_ext).

Además, los dos periodistas de la pieza (De Pablo y Zurita) cuentas con sus propias páginas web (http://www.ofeliadepablo.es/y http://javierzurita.com/). En ambas páginas explican su trabajo fotoperiodístico y lo difunden. Ambos tienen un apartado específicos para piezas similares a Spain gets angry, donde combinan diferentes géneros y medios de expresión: banda sonora (diálogos, narración, sonido ambiente), fotografía, vídeo y textos (incluyendo data journalism).

-Drillable (que fomenta la profundización): Se trata de completar la historia, permitir que el lector profundice a través de enlaces que les lleven a otras narraciones, datos, o noticias y reportajes relacionados. En definitiva, que el lector sea también un investigador guiado por su curiosidad y ánimo de conocer más de la historia o de acontecimientos similares (Moloney, 2011: 64). Así, la pieza tendrá enlaces, hipervínculos y una carga, más o menos amplia, de informaciones alternativas y contextuales al que el lector puede acceder.

En el ejemplo que aquí se analiza, bajo el vídeo de Zurita y de Pablos, se encuentra otro enlace titulado "Madrid protesters force police to retreat in clashes over treatment of immigrants" situado bajo la etiqueta "More on this story". Se trata de un artículo sobre otras manifestaciones ciudadanas en Madrid, lo que ayuda a completar la historia primera. También aparece el enlace a "Related videos" donde se incluye un vídeo sobre las protestas del pueblo argentino contra su gobierno y permite al lector comparar situaciones en el ámbito internacional.

-Continuous and Serial (continuidad del estilo editorial): Con serial se refiere a la publicación en partes o capítulos de las piezas, o cómo las diferentes perspectivas de una historia pueden contarse de forma seriada. Por su parte, el rasgo continuous difiere ligeramente de las narrativas de ficción. En el ámbito del periodismo se trata sobre todo de construir las piezas con la colaboración de varios periodistas. También hace referencia a la coherencia y continuidad editorial. En este caso se trata de la unión de dos periodistas especializados en la imagen. De hecho, ambos son fotógrafos y su archivo visual y su forma habitual de registrar la realidad les han permito crear una pieza de atractivo visual. Aunque Moloney sugiere que la mejor fórmula para conseguir esta continuidad es el trabajo bien coordinado de un grupo de periodistas (Moloney, 2011: 71), admite que la unión profesional de individualidades bien organizadas puede dar 
el mismo resultado. En el caso analizado estaríamos en el primer ejemplo, es decir, un grupo de periodistas bien organizado, aunque reducido, pero la posibilidad del trabajo online permite la descentralización y la desubicación de los grupos de trabajo.

-Diverse and Personal in Viewpoint (Subjectivity) (aportación de diferentes puntos de vista): Conectado con el punto anterior, se trata, por un lado, de que cada periodista aporte un discurso. Aquí Moloney hacer referencia a la imposibilidad de la completa objetividad y apela a la honestidad del profesional. Diríamos que al periodista se le presupone este rasgo en su trabajo, como al médico su interés natural en la curación del paciente. Además, incluye en este viewpoint la participación de los lectores a través de sus opiniones y comentarios. Es decir, no solo el discurso se construye con la multiplicidad de narrativas (entrevistas, textos, videos, etc.) sino que a ellas se suma la participación de un lector-periodista (periodismo ciudadano) que ayuda a completar datos. Es aquí donde se puede hablar de subjetividad. Aunque en la página de The Guardian no hay un espacio habilitado para los comentarios de los lectores, la pieza se ha difundido de "click a click" y diferentes lectores lo han embebido en sus blogs, webs u otras plataformas donde sí realizan comentarios. Entre los lugares donde podemos encontrar la pieza se encuentran el twitter del australiano Earl Edward de Vere (2011) o IndyMedia Ireland (2011), entre otros.

-Immersive (inmersión): Se trata de conseguir que el lector se detenga en la historia que el periodista le cuenta y que le atrape con un lazo invisible construido con referencias, no solo al hecho, sino también a otros datos sensoriales, por ejemplo un olor:

"Former Chicago Tribune correspondent Laurie Goering explained to me years ago that an old editor she respected once asked her to "tell me how it smelled" to get her to think of making those mental images in the reader's mind" (Personal communication, 1995). (Moloney, 2011: 80-81)

Una idea en la que Moloney hace especial hincapié y a la que dedica ejemplos y reflexiones es la de un nuevo producto: el videojuego periodístico. Se trata de un grupo de profesionales que unen investigación y tecnología para desarrollar juegos o entornos multimedia para que el lector-jugador tenga una experiencia global y verdaderamente inmersiva (Moloney, 2011: 81-85). Los mundos creados (por ejemplo en la plataforma Second Life) llevan al usuario a Guantánamo o a una fila de reparto de comida, de tal modo que entiende "desde dentro" la problemática de ese problema. Así, no solo conoce los datos, también los sonidos y toda la experiencia es vivida en primera persona.

Moloney trae aquí otras reflexiones como la vivencia que hace la narración "suya" a través de la conexión con sus emociones. Esta idea está conectada con el periodismo de datos y cómo se está expandiendo gracias a la diversidad de opciones de visualizaciones en red (juegos, mapas conceptuales interactivos, audios, etc.). Los autores Flores y Salinas (2012: 9) relacionan de forma directa el periodismo de datos y la narrativa transmedia. Se trata de un soporte mutuo o simbiosis en el que el discurso multimedia y transmediático se apoya en recursos en red tales como mashups. Aunque Spain gets angry no presenta este periodismo de datos, sí lo podemos ver en otras pie- 
zas similares de los mismos periodistas como The invisible genocide para el New York Times. La inmersión es una herramienta ya usada por el periodismo de investigación y que en las Reglas quebrantables de Kramer (2001) ya se recoge y defiende.

-Extractable (extracción): Este rasgo se refiere a qué o cómo el lector puede extraer algo útil de la pieza periodística para aprovechar en su vida, por eso, este punto toca diferentes aspectos. No solo se refiere a que obtengamos información necesaria para vivir en comunidad (Moloney, 2011: 86), sino a aspectos como los juegos o los regalos que sacan los periódicos con sus ediciones dominicales o especiales. Pero aspecto está íntimamente relacionado con el siguiente que completa su sentido (inspiring action). En todo caso, pensando en el $15 \mathrm{M}$ y la información que aporta el vídeo podríamos hablar de que la pieza permite extractar informaciones como los mensajes de las pancartas, eslóganes de la manifestación, etc., y, al mismo tiempo, los datos aportados, sobre todo a través de las entrevistas, ayudan al usuario a conocer datos de fuentes primarias lo que le permite tomar decisiones en su entorno social e incluso privado.

- Inspiring to Action (mover al lector hacia la acción, para tomar partido): Considero que los discursos transmediáticos, probablemente gracias a los puntos anteriores, son más propicios a conseguir cambios en las personas: en sus acciones diarias o en su toma de decisiones y es ese aspecto el que permite hablar de la "extracción" de una decisión desde un discurso dado y de motivar al lector para la acción. Moloney cita a Jacob Riis como ejemplo, no solo de inmersión, sino también de activador de cambios (Moloney, 2011: 91). El Periodismo como profesión ha estado motivado desde sus inicios por una meta: mejorar la sociedad y ayudar al ciudadano. Así, si pensamos en Spain gets angry y cómo nos ayuda a ahondar en las razones del enfado y decepción ciudadanas y comprendemos sus motivos, entonces el lector/espectador puede sentirse (con)movido a apoyar el movimiento, sus objetivos y tomar la decisión de salir a la calle o realizar otro tipo de acción solidaria. Con piezas que ayudan a conocer y comprender el contexto de un acontecimiento el ciudadano comprende más profundamente las motivaciones de las personas y puede participar de ellas. Esto también sucede con el Periodismo Literario.

-Built in Real Worlds (profundización en los mundos colaterales): El periodismo no tiene como finalidad construir mundos nuevos (como ocurre en la ficción) porque ya existen, pero sí puede esforzarse en dar voz a diferentes actores de un hecho y no tratar de simplificar el acontecimiento hasta convertirlo en algo espontáneo, sin causas o historia anterior o un futuro. Moloney ilustra esta idea con el documental Restrepo (2010) y la siguiente cita de su director Tim Hetherington:

"There's a great emphasis in war reporting on capturing the actual "bang-bang" fighting of war - and many reporters feel that any work would be incomplete without a sense of this "action." We were no different, but because there was an incredible amount of fighting going on in the Korengal Valley, recording the actual firefights got quite boring. What was infinitely more interesting and revealing was how the soldiers carried on in these situations. People who haven't experienced war inevitably base their understanding of it [on] the mediated versions of news or Hollywood. These representations are often limited and can't quite reveal the humor, boredom, and confusion inherent in combat. It's something we felt was important to represent" (Moloney, 2011: 89-90) 
Para conocer una historia, un acontecimiento o un momento histórico y, además, comprenderlo, no basta con preguntar al protagonista más destacado, sino contar con los testimonios de otras personas que viven o sufren el hecho, así se completa la visión en $360^{\circ}$. Leibovitz (2006) afirma que las cosas más interesantes, los momentos claves, ocurren cuando nadie mira. Este es también el caso de Spain gets angry donde se entrevista a tres personas sin puestos destacados o poder para tomar decisiones. Las entrevistas se realizan a una madre soltera, un bombero y una joven perteneciente al movimiento $15 \mathrm{M}$. No son personajes públicos, no tienen coches oficiales ni se reúnen con los políticos, pero son ejemplos de lo que sucede en las capas sociales que más sufren la crisis global y sus testimonios nos permiten conocer más de cerca la situación de millones de españoles, sus razones profundas, su voz y sus emociones.

\section{Conclusiones}

Con lo aportado por Moloney se entienden las oportunidades que la narrativa transmedia aporta al periodismo y a su crisis estructural. Además, también aumenta exponencialmente la democratización de la información que ya no está intervenida por medios verticales sino que se expande sin control institucional. También permite la renovación y el fortalecimiento del periodismo de investigación, el data journalism, la inmersión del lector, la difusión masiva y la ruptura con los fuertes lazos de la monopolización editorial de los medios. Todos estos rasgos vienen apoyados y refuerzas a su vez el open data y Marta Franco ya lo califica como "clave para el periodismo digital" (23/01/2013). Moloney afirma en este sentido "Transmedia storytelling techniques would not only reach wider publics, but provide deeper engagement with the story being told, more context and a conversation about the issue rather than just a lecture." (2011: 92).

Las posibilidades transmediáticas y multimedia no son un catálogo limitado que el periodista debe usar al completo. Es un gran universo de posibilidades que deben adaptarse y elegirse dependiendo del tema, de la naturaleza del reportaje y del propio periodista. Además, el profesional debe recordar que la historia es lo primero y lo más importante (Looney, 30/6/2013). No solo el periodista ha de elegir la fórmula narrativa y multimedia (diseño web, hipertexto, comic, videojuego, etc.) sino incluso el medio más adecuado para su difusión. Como afirma Sam Ford (2007) no se trata de que un solo profesional haga toda la cadena de producción (investigue, tome fotos, redacte, publique, suba el vídeo, etc.) sino de encontrar la mejor plataforma para la historia. Como explica Marta Franco debe ser un "equipo de redactores que dominen el mundo multimedia y entiendan las reglas del juego de la comunicación digital" $(30 / 11 / 2012)$. A esto se debe añadir que el trabajo en equipo es fundamental para hacer piezas completas e inmersivas y, por supuesto, el grupo debe tener un profundo archivo visual personal que le permita, apelando a la historia de las imágenes, una comunicación profunda con el lector. De esta forma, las piezas serán capaces de acercarse a la complejidad de nuestro mundo y también de las historias contadas.

Las empresas mediáticas ya están comenzando a ver las posibilidades que ofrece la narrativa transmediática unida al periodismo de investigación. Un buen ejemplo internacional de esta tendencia creciente es el New York Times. Este medio ponía en 
marcha en julio de 2013 un cambio profundo en su redacción encaminado a crear nuevas secciones, principalmente para su plataforma online. Sabedores de la potencia mediática que tienen (según los datos que aportan (Editorial: 12/7/2013) la mitad de sus visitantes lo hacen desde plataformas móviles), aprovechan para movilizar a sus mejores trabajadores y definir nuestras estrategias periodísticas y discursivas. La base desde la que (re)nace el New York Times es la pieza Snow Fall, presentada en los primeros apartados de esta investigación y que aporta no solo un discurso transmediático, sino también la actualización de viejos géneros como el periodismo literario o el periodismo de datos unida a una estrategia periodística de inmersión que lleva al lector a adentrarse en una historia de $360^{\circ}$ y apuesta por la lectura profunda. Lo que podríamos denominar siguiendo los neologismos actuales: el slow journalism.

El periodismo más visual realizado en el siglo XXI se ha caracterizado por incorporar a sus fórmulas narrativas técnicas procedentes de otras ramas de la comunicación, como por ejemplo las del lenguaje fotográfico más creativo (fotoperiodismo literario, fotoperiodismo con teléfono móvil,...). Ahora, la narrativa transmediática viene a incorporarse a una nueva generación de periodistas que tienen en cuenta no solo los nuevos formatos de publicación o difusión, sino también a los mismos lectores multiplataforma que les ayudan en la democratización de la información y en la toma de decisiones. Todo el conjunto de pequeñas revoluciones y cambios es lo que Roger Fidler denominó mediamorfosis (1998) es ahora más democrática que nunca.

\section{Referencias bibliográficas}

BEESON, Michael (2005): "Cross-Media Narrative", en: http://ncca.bournemouth.ac.uk/gallery/view/45/Cross-Media_Narrative

BRANCH, John (2012): "Snow Fall: The Avalanche at Tunnel Creek". The New York Times Projects: http://www.nytimes.com/projects/2012/snow-fall/

CAMPALANS, Carolina (2012): "Reseña" del libro de Renó, D. y Flores, J. (2012): Periodismo transmedia. Reflexiones y técnicas para el ciberperiodista desde los laboratorios de medios interactivos. Madrid, Fragua, en Palabra Clave, vol. 15, $\mathrm{n}^{\mathrm{o}}$ 3, pp. 733-735.

CARRIÓN, Jorge (2012): "El nuevo nuevo periodismo" en: $\mathrm{http}$ ://jorgecarrion.com/2012/12/21/el-nuevo-nuevo-periodismo/

CARRIÓN, Jorge (2013): "Periodismo expandido" en VI Jornadas de Periodismo y Literatura (conferencia). Universidad San Jorge, Zaragoza, 8 de mayo.

DE PABLO, Ofelia: Periodista y documentalista. Web con sus trabajos multimedia http://www.ofeliadepablo.es/multimedia

DE VERE, Earl Edward (2011): Guardian: Spain gets angry (21 de junio). En: https://twitter.com/EdDeVere/status/83221406199975936

FIDLER, Roger (1998): Mediamorfosis. Buenos Aires, Ediciones Granica.

FLORES, Jesús y SALINAS, Cecilia (2012): "Sinergias en la construcción del Nuevo Periodismo derivadas del Data Journalism y el Transmedia Journalism", en III Congreso Internacional de Comunicación 3.0. Salamanca: http://www.2ip.es/wpcontent/uploads/2013/01/sinergias_construccion_nuevoperiodismo.pdf 
FORD, Sam (2007): "Transmedia Journalism: A Story-Based Approach to Convergence", 5 de abril, en Futures of Entertaiment: http://www.convergenceculture.org /weblog/2007/04/transmedia_journalism_a_storyb.php

FRANCO, Marta (2013): "Un nuevo camino para la narración multimedia", en el blog Marta Franco: Ideas para comunicar en la nube, 23 de enero: http://martafranco.es/un-nuevo-camino-para-la-narracion-multimedia/

FRANCO, Marta (2013): "Los nuevos profesionales en el periodismo digital", en el blog Marta Franco: Ideas para comunicar en la nube", 20 de febrero: http://martafranco.es/los-nuevos-profesionales-en-el-periodismo-digital/

INDYMEDIA IRELAND (2011, Web): \#SpanishRevolution comes to Greece: \#M25Gr = \#GreekRevolution, 27 de mayo, en: http://www.indymedia.ie/article $/ 99876$

JENKINS, Henry (2003): "Transmedia Storytelling. Moving characters from books to films to video games can make them stronger and more compelling", en http://www.technologyreview.com/news/401760/transmedia-storytelling/

JENKINS, Henry (2007): “Transmedia Storytelling 101", en: http://henryjenkins.org/2007/03/transmedia_storytelling_101.html

JENKINS, Henry (2009): "The Revenge of the Origami Unicorn: Seven Principles of Transmedia Storytelling", 12 de diciembre, en: http://henryjenkins.org /2009/12/the_revenge_of_the_origami_uni.html

KRAMER, Mark (2001): "Reglas quebrantables para periodistas literarios". El Malpensante, $\mathrm{n}^{\mathrm{o}} 32$, pp. 73-85. También en: http://elmalpensante.com/print_contenido.php?id $=2349$

LEIBOVITZ, Barbara (2006): "Annie Leibovitz: una vida a través de una cámara". DVD, 83', versión original en inglés subtitulada al español. New York, Thirteen/WNET.

LOONEY, Margaret (2013): "5 Tips for Transmedia Storytelling", 30 de junio, en: http://www.pbs.org/mediashift/2013/01/5-tips-for-transmedia-storytelling030

MICHAEL (2005): Cross-Media Narrative. Bournemouth University, en: http://ncca.bournemouth.ac.uk/gallery/view/45/Cross-Media_Narrative

MOLONEY, Kevin (2012): “Transmedia Journalism in 499 Words", 6 de noviembre, Transmedia Journalism, en: http://transmediajournalism.org/2012/11/06/transmedia-journalism-in-499-words/

MOLONEY, Kevin (2012): "Transmedia Journalism as a Post-Digital Narrative", 2 de noviembre, en Running head: Transmedia Journalism as a Post-Digital Narrative. University of Colorado.

MOLONEY, Kevin (2012): “Transmedia Journalism: A new storytelling strategy and a blog to go with it", 8 de agosto, en Transmedia Journalism: http://blog.kevinmoloney.com/?p=1073

MOLONEY, Kevin (2011): “Transmedia Journalism in Principle”, 23 de noviembre, en Transmedia Journalism: http://transmediajournalism.org/2011/11/23/transmedia-journalism-in-principle/ 
MOLONEY, Kevin (2011): Porting Transmedia Storytelling to Journalism. Thesis (In Partial Fulfillment of the Requirements for the Degree Master of Arts. Advisor: Dr. Adrienne Russell). Faculty of Social Sciences. University of Denver, en: http://www.kevinmoloney.com/Transmedia_Journalism.pdf

PERESS, Gilles y Ritchin, Fred (1996): Bosnia: Uncertain Paths To Peace. En: http://www.pixelpress.org/bosnia/intro.html

SCOLARI, Carlos (2009): "Narrativas transmediáticas: breve introducción a un gran tema", 15 de mayo, en Hipermediaciones. Conversaciones sobre la comunicación digital interactiva: http://hipermediaciones.com/2009/05/15/narrativas-transmediaticas/

SCOLARI, Carlos (2013): "Evolución de los medios: las relaciones peligrosas”, 16 de abril, en http://hipermediaciones.com/2013/04/16/evolucion-de-los-medios-las-relaciones-peligrosas/\#more-2959

THE HUFFINGTONPOST.COM (2013): "New York Times Shake-Up: Sam Sifton Out As National Editor", artículo editorial, 12 de julio en: http://www.huffingtonpost.com/2013/07/12/new-york-times-changes-sam-sifton_n_3587165.html

ZURITA, Javier: Fotoperiodista. Web con sus trabajos multimedia: http://javierzurita.com/multimedia

ZURITA, Javier y DE PABLO, Ofelia (2011): Spain gets angry, 20 de junio: http:/www.theguardian.com/world/video/2011/jun/20/spain-gets-angry-m15-protest-video 